\title{
Hybrid 1D/2D heterostructure with electronic structure engineering toward high-sensitivity and polarization-dependent photodetector
}

\author{
Yuchen Zhou ${ }^{1}$, Lixiang Han ${ }^{1}$, Qiqi Song ${ }^{1}$, Wei Gao ${ }^{2}$, Mengmeng Yang ${ }^{1}$, Zhaoqiang Zheng ${ }^{*}$, Le Huang ${ }^{1}$, \\ Jiandong $\mathrm{Yao}^{3}$ and Jingbo $\mathrm{Li}^{2,4,5^{*}}$
}

\begin{abstract}
The widespread application of photodetectors has triggered an urgent need for high-sensitivity and polarization-dependent photodetection. In this field, the two-dimensional (2D) tungsten disulfide ( $\left.\mathrm{WS}_{2}\right)$ exhibits intriguing optical and electronic properties, making it an attractive photosensitive material for optoelectronic applications. However, the lack of an effective built-in electric field and photoconductive gain mechanism in $2 \mathrm{D} \mathrm{WS}$ impedes its application in high-performance photodetectors. Herein, we propose a hybrid heterostructure photodetector that contains 1D Te and 2D WS 2 . In this device, 1D Te induces in-plane strain in $2 \mathrm{D} \mathrm{WS} \mathrm{S}_{2}$, which regulates the electronic structures of local $\mathrm{WS}_{2}$ and gives rise to type-II band alignment in the horizontal direction. Moreover, the vertical heterojunction built of $2 \mathrm{D} \mathrm{WS}$ and $1 \mathrm{D}$ Te introduces a high photoconductive gain. Benefiting from these two effects, the transfer of photogenerated carriers is optimized, and the proposed photodetector exhibits high sensitivity (photoresponsivity of $\sim 27.7 \mathrm{~A} \mathrm{~W}^{-1}$, detectivity of $9.5 \times 10^{12}$ Jones, and short rise/ decay time of $19.3 / 17.6 \mathrm{~ms}$ ). In addition, anisotropic photodetection characteristics with a dichroic ratio up to 2.1 are achieved. This hybrid 1D/2D heterostructure overcomes the inherent limitations of each material and realizes novel properties, opening up a new avenue towards constructing multifunctional optoelectronic devices.
\end{abstract}

Keywords: hybrid heterostructure, electronic structure engineering, photodetector, anisotropic photodetection

\section{INTRODUCTION}

Due to the exotic structure, electric and optical properties, twodimensional (2D) transition metal dichalcogenides (TMDs) have shown high potential in next-generation photoelectronic devices [1-3]. Among the 2D TMD family, 2D tungsten disulfide $\left(\mathrm{WS}_{2}\right)$ holds the advantages of the highest predicted carrier mobility and comparatively wide bandgap, making it widely favored in the construction of high-performance optoelectronic devices [46]. However, individual $2 \mathrm{D} \mathrm{WS}_{2}$ lacks an effective built-in electric field and a photoconductive gain mechanism, which cannot rapidly separate the photoexcited carriers for repeat circulation in the external circuit $[7,8]$. Therefore, the performance of the individual $\mathrm{WS}_{2}$-based photodetectors is generally limited [9-11]. In addition, modern optoelectronic applications increasingly require polarization-dependent photodetection [12-14], which cannot be achieved by $2 \mathrm{D} \mathrm{WS}_{2}$.

To address these issues and construct optoelectronic devices that simultaneously possess high photoresponse and polarization-dependent photosensitivity, many technologies have been developed. On one hand, the introduction of local strain can redistribute the charge carriers and thus tune the local band structure, forming intramolecular homojunctions $[15,16]$. As a result, built-in electric fields are formed, which can accelerate the separation of photoexcited carriers and improve the speed of the photoelectric devices [17]. On the other hand, on account of the dangling-bond-free surface of 2D TMDs, van der Waals heterojunctions (vdWHs) can be easily achieved without considering the lattice matching constrains. Such vdWHs can not only overcome the inherent limitations of each material, but also achieve novel properties through appropriate combination of materials with complementary properties. For example, Ye et al. [18] reported a photodetector based on a black phosphorus (BP)/WSe $\mathrm{WdWH}_{2}$. Through employing BP as the photogate and $\mathrm{WSe}_{2}$ as the conductive channel, the device exhibits high responsivity, as well as pronounced polarization-dependent photosensitivity.

To take full advantage of these technologies, herein, we demonstrate a hybrid $1 \mathrm{D} / 2 \mathrm{D}$ heterostructure photodetector made of a Te nanowire (NW) and a $\mathrm{WS}_{2}$ nanoflake. As a p-type semiconductor, Te has attracted tremendous interest due to its competitive features, such as high carrier mobility, anisotropic crystal structure and good air stability [19-21]. In addition, the 1D Te NW with anisotropy in geometry provides an additional degree of freedom in device construction. In this design, an exfoliated $\mathrm{WS}_{2}$ nanoflake is dry-transferred onto a Te NW, which induces in-plane strain in $\mathrm{WS}_{2}$. This strain regulates the electronic structures of local $\mathrm{WS}_{2}$. Through reasonable voltage regulation, two continuous type-II band alignments are formed

\footnotetext{
${ }^{1}$ Guangdong Provincial Key Laboratory of Information Photonics Technology, School of Materials and Energy, Guangdong University of Technology, Guangzhou 510006, China

${ }^{2}$ Institute of Semiconductors, South China Normal University, Guangzhou 510631, China

${ }^{3}$ State Key Laboratory of Optoelectronic Materials and Technologies, Nanotechnology Research Center, School of Materials Science \& Engineering, Sun Yatsen University, Guangzhou 510275, China

${ }^{4}$ Guangdong Provincial Key Laboratory of Chip and Integration Technology, Guangzhou 510631, China

${ }^{5}$ State Key Laboratory for Superlattices and Microstructures, Institute of Semiconductors, Chinese Academy of Sciences, Beijing 100083, China

*Corresponding authors (emails: zhengzhq5@mail2.sysu.edu.cn (Zheng Z); jbli@semi.ac.cn (Li J))
} 
in the horizontal direction of $\mathrm{WS}_{2}$, which can accelerate the separation of photogenerated carriers and suppress dark current. Besides, the vertical heterojunction built of $\mathrm{WS}_{2}$ and Te introduces photoconductive gain, thereby extending the lifetime of photoexcited carriers. Based on these synergetic effects, a high photoresponsivity of $\sim 27.7 \mathrm{~A} \mathrm{~W}^{-1}$, an excellent detectivity of 9.5 $\times 10^{12}$ Jones, and a short rise/decay time of $19.3 / 17.6 \mathrm{~ms}$ are demonstrated. These values are much better than those of the individual $\mathrm{WS}_{2}$ device. Furthermore, anisotropic photodetection characteristics with a dichroic ratio up to 2.1 are achieved. This work provides an effective pathway towards constructing highperformance optoelectronic devices.

\section{EXPERIMENTAL SECTION}

\section{Device construction}

First, the Te NWs were prepared by a physical vapor deposition (PVD) method (the detailed process was described in Supplementary information Note S2). Then, one Te NW was transferred onto a clean $\mathrm{SiO}_{2} / \mathrm{Si}$ substrate with the assistant of polyvinyl alcohol (PVA). Sequentially, a multilayer $\mathrm{WS}_{2}$ (M$\mathrm{WS}_{2}$ ) nanoflake obtained by mechanical peeling from bulk crystal was dry transferred onto a Te NW with the help of a 3D positioning platform (Shanghai OnWay Technology Co., Ltd). Later, for the fabrication of $\mathrm{WS}_{2}, \mathrm{Te} \mathrm{NW}$ and $\mathrm{M}-\mathrm{WS}_{2} /$ Te devices, corresponding electrodes were patterned via photolithography (a maskless photolithography machine of TuoTuo Technology Co., Ltd). Next, Ti/Au (10/50 nm) electrodes were deposited using electron beam evaporation (EB-400S, Ecopia), followed by a lift-off process. Finally, the whole devices were annealed at $150^{\circ} \mathrm{C}$ for $3 \mathrm{~h}$ to ensure close contact at the interface of each component.

\section{Characterizations}

The instruments used to observe the morphology of the samples are a microscope (BA310Met, Motic) and a scanning electron microscope (SEM) system (LYRA 3 XMU, Tescan). Raman spectra and PL spectra excited by a 325-nm laser or 532-nm laser were collected by a confocal microscope (LabRAM HR Evolution, HORIBA Jobin Yvon). A Te NW was transferred onto the copper grid for transmission electron microscopy (TEM) (Talos F200S, FEI) characterization. Atomic force microscopy (AFM) and Kelvin probe force microscopy (KPFM) measurements were carried out on a scanning probe microscope (Dimension FastScan, Bruker). Electrical and optoelectronic characteristic characterizations of all devices were performed in the probe station integrated with a Keithley $2614 \mathrm{~b}$ Source table. Transient photocurrent response was recorded using a digital oscilloscope (DPO 4102B, Tektronix). A laser with a wavelength of $405 \mathrm{~nm}$ and a bromine tungsten lamp were used as the light sources. The incident spot was a circle with a radius of $3 \mathrm{~mm}$, which covered the whole photosensitive region. Polarization-dependent photoresponse was characterized through rotating the half-wave plate in the light path below the polarizer to adjust the polarization of the incident light.

\section{RESULTS AND DISCUSSION}

The fabrication procedures for the $\mathrm{WS}_{2} / \mathrm{Te}$ device are shown in Fig. 1a. The Te NWs were synthesized on a $\mathrm{SiO}_{2} / \mathrm{Si}$ substrate through the PVD method. Then, one Te NW was transferred on
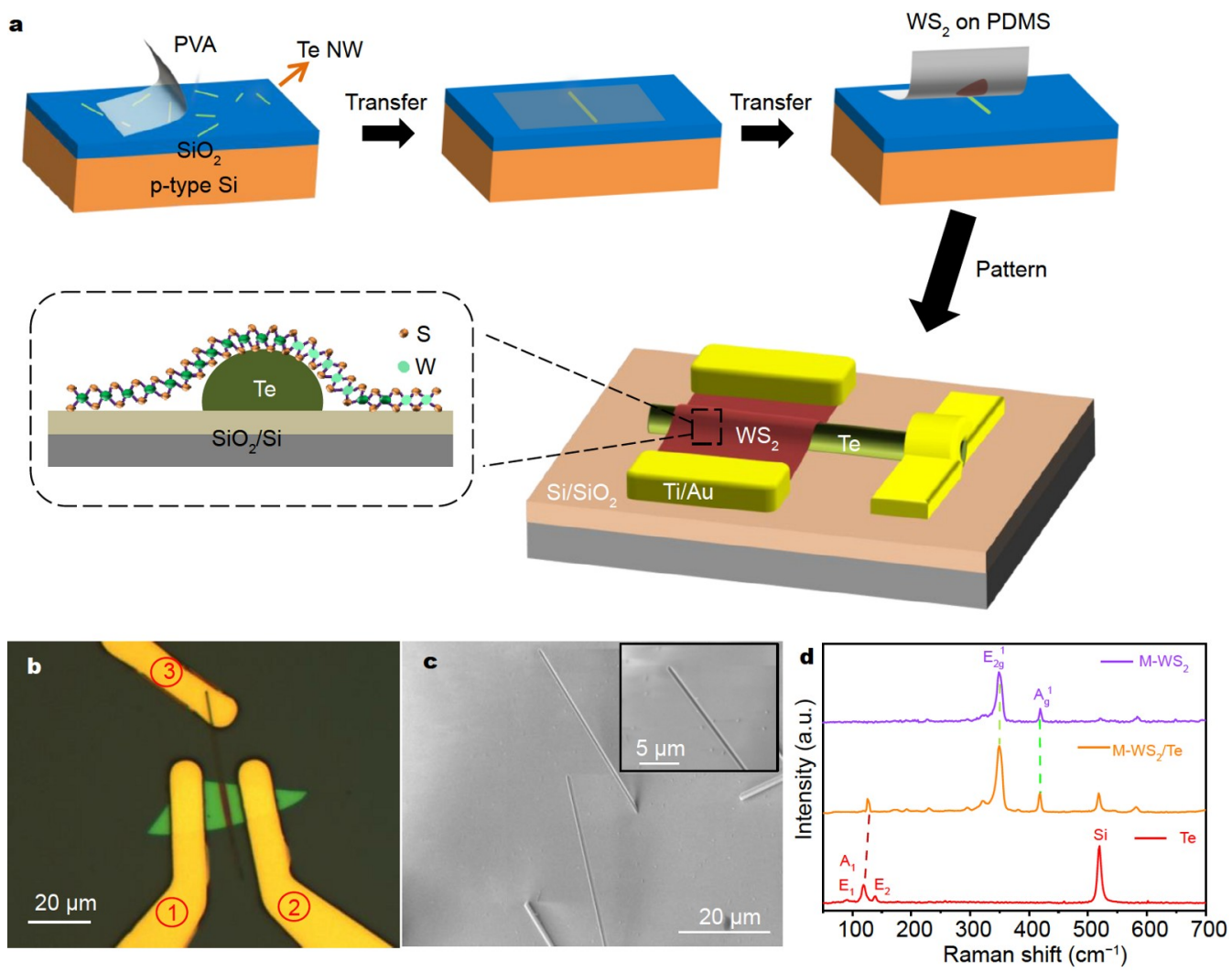

Figure 1 (a) Schematics showing the fabrication process of the $\mathrm{M}-\mathrm{WS}_{2} / \mathrm{Te}$ device and the local atomic structure. (b) Optical microscopy image of the M$\mathrm{WS}_{2} / \mathrm{Te}$ device. The red numbers indicate the three electrodes. (c) SEM image of the Te NWs grown by the PVD method. Inset: enlarged SEM image of a single Te NW. (d) Raman spectra of $\mathrm{M}-\mathrm{WS}_{2}$, Te NW and the M-WS $2 / \mathrm{Te}$ overlapping area utilizing a 532-nm excitation laser. 
a clean $\mathrm{SiO}_{2} / \mathrm{Si}$ substrate, followed by the capping of an $\mathrm{M}-\mathrm{WS}_{2}$ nanoflake, which thus formed a hybrid $1 \mathrm{D} / 2 \mathrm{D}$ heterostructure $\left(\mathrm{M}-\mathrm{WS}_{2} / \mathrm{Te}\right)$. The $\mathrm{Ti} / \mathrm{Au}$ electrodes were patterned and deposited using photolithography and lift-off techniques. Fig. 1b shows an optical microscopy image of the actual device obtained by the above method. It can be seen that the device is clean and tidy, and the $M-W_{2}$ is tightly attached to the Te NW. The typical SEM image of the fabricated Te sample is provided in Fig. 1c. The Te NWs grow obliquely on the substrate, which minimizes the impact from the substrate and offers a clean surface. In addition, the calculated aspect ratio of the Te NWs is more than 150, revealing the anisotropy of Te sample. Then, Raman scattering measurements were adopted to further investigate the $\mathrm{M}-\mathrm{WS}_{2} /$ Te heterostructure. As shown in Fig. 1d, the purple, orange and red lines represent the Raman spectra of the $\mathrm{M}-\mathrm{WS}_{2}, \mathrm{M}-\mathrm{WS}_{2} / \mathrm{Te}$ and Te NWs, respectively. The peaks located at 348.7 and $419.1 \mathrm{~cm}^{-1}$ can be indexed to the $\mathrm{E}_{2 \mathrm{~g}}^{1}$ and $\mathrm{A}_{\mathrm{g}}^{1}$ modes of $\mathrm{WS}_{2}$, respectively [22]. The Te NWs have three characteristic peaks at $89.6,119.1$ and $139.3 \mathrm{~cm}^{-1}$, which can be assigned to $E_{1}, A_{1}$ and $E_{2}$ vibration modes, respectively [23]. In $\mathrm{M}-\mathrm{WS}_{2} / \mathrm{Te}$, the $\mathrm{A}_{1}$ peak of Te NWs exhibits slight red shift, while the $E_{1}$ and $E_{2}$ peaks have disappeared. Besides, the $E_{2 g}^{1}$ peak of $\mathrm{M}-\mathrm{WS}_{2}$ shows obvious quenching and blue shift (Fig. S1). These phenomena are due to the bending deformation of $\mathrm{M}-\mathrm{WS}_{2}$ which introduces strain in $\mathrm{M}-\mathrm{WS}_{2}[24,25]$. Next, TEM was used to characterize the Te NWs. The high-angle annular dark-field (HAADF) image in Fig. S2a shows that the size of Te NW is uniform. The energy dispersive spectroscopy (EDS) spectrum in Fig. S2b shows that the sample contains only Te element, indicating that the prepared Te NWs are free of impurities. The element mapping image in Fig. S2c reveals that Te is evenly distributed throughout the entire wire, corroborating the compositional uniformity of the Te NW [26]. Subsequently, X-ray diffraction (XRD) of the Te NWs was characterized and the result is shown in Fig. S2d. All diffraction peaks are consistent with the standard card of Te (PDF 036-1452). The salient peaks located at $23.1^{\circ}$ and $27.6^{\circ}$ can be indexed to (100) and (101) lattice planes, respectively. All these results confirm the high quality of the Te NWs.

In order to investigate the carriers' separation behavior between Te and $\mathrm{M}-\mathrm{WS}_{2}$, photoluminescence (PL) characterization was performed. As shown in Fig. 2a, there are two conspicuous peaks at 524 and $640 \mathrm{~nm}$ for the $\mathrm{M}-\mathrm{WS}_{2}$, which are attributed to the exciton absorption in the direct gap of the $\mathrm{K}$ valley in the Brillouin zone. Due to the spin-orbit coupling, the valence band minimum splits, which leads to the separation of these peaks [27]. The PL peaks of the $\mathrm{M}-\mathrm{WS}_{2} / \mathrm{Te}$ show obvious quenching and red shift, which reveals the existence of effective separation of photogenerated carriers in the overlapping region

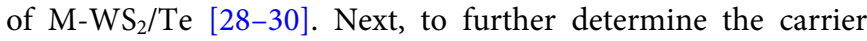
transfer process, electronic structures of flat $\mathrm{M}-\mathrm{WS}_{2}$ and $\mathrm{M}-\mathrm{WS}_{2}$ with varied degrees of deformation were calculated within the Perdew-Burke-Ernzerhof (PBE) form (the detailed calculation process is described in Supplementary information Note S1).
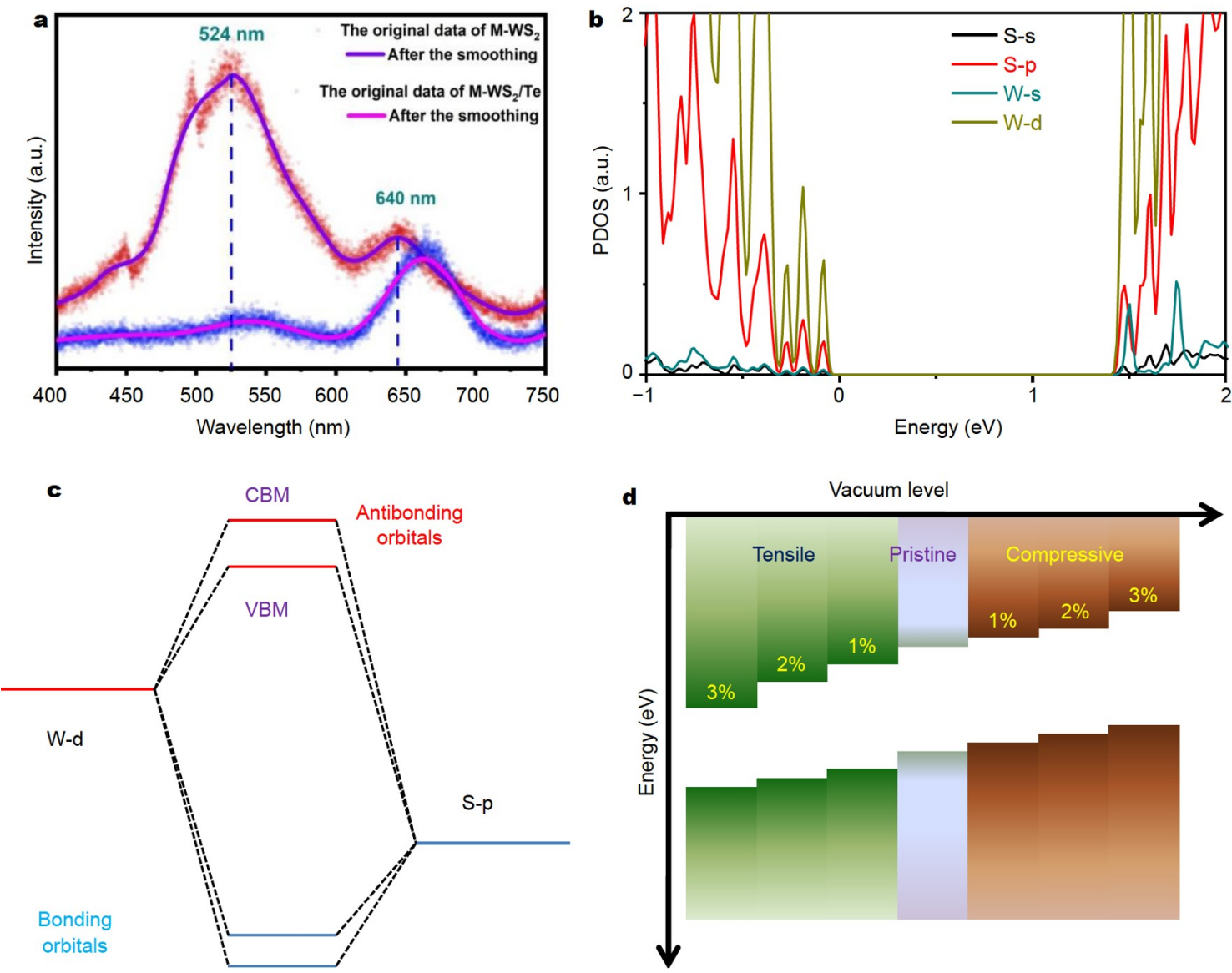

Figure 2 Strain analysis of the M-WS 2 . (a) PL spectra of $\mathrm{M}-\mathrm{WS}_{2}$ and $\mathrm{M}-\mathrm{WS}_{2} / \mathrm{Te}$ under excitation of 325-nm laser. (b) Calculated PDOS of the pristine M$\mathrm{WS}_{2}$. (c) Orbital coupling model of M-WS . (d) The evolution of the CBM and VBM positions of M-WS ${ }_{2}$ under different deformations. 
Fig. S3a presents the calculated band structure of the pristine $\mathrm{WS}_{2}$, and a direct band gap located at the K point is observed. To analyze the orbital coupling, partial density of states (PDOS) of the $\mathrm{WS}_{2}$ has also been calculated and shown in Fig. 2b. It is shown that both the valence band maximum (VBM) and conduction band minimum $(\mathrm{CBM})$ are determined by the hybridization of $\mathrm{W}-\mathrm{d}$ and $\mathrm{S}-\mathrm{p}$ orbitals. Fig. 2c shows the corresponding orbital coupling model. It is observed that $\mathrm{W}-\mathrm{d}$ and S-p form the bonding orbitals and antibonding orbitals, and the latter determine the positions of CBM and VBM. Actually, the degree of orbital coupling is highly sensitive to the amount of strain [16]. In the $\mathrm{M}-\mathrm{WS}_{2} / \mathrm{Te}$ device, both tensile and compressive effects are introduced into $\mathrm{M}-\mathrm{WS}_{2}$ through bending. The upper surface of $\mathrm{M}-\mathrm{WS}_{2}$ shows the largest tensile deformation, while the bottom surface shows the largest compressive deformation. For the convenience of calculation and analysis, we just consider the impacts of the above two extreme conditions in the deformation of $\mathrm{M}-\mathrm{WS}_{2}$. Fig. S3b, c present the typical band structures of $\mathrm{M}-\mathrm{WS}_{2}$ under large deformation. Under compression, both of the VBM (at the $\Gamma$ point) and the CBM (between the $\mathrm{K}$ and $\Gamma$ points) move upward, and the band gap increases. In contrast, under tension, the VBM and CBM move downward, and the band gap decreases. Considering the redshift of PL peaks, it is believed that the effect of tensile strain plays a leading role in the deformation of $\mathrm{M}-\mathrm{WS}_{2}$. Fig. $2 \mathrm{~d}$ presents the calculated energy band arrangement of $\mathrm{M}-\mathrm{WS}_{2}$ under varied degrees of deformation. Since the energy bands move in the same direction, intramolecular homojunctions with type-II band alignments are formed between the pristine $\mathrm{M}-\mathrm{WS}_{2}$ and the deformed $\mathrm{M}-\mathrm{WS}_{2}$, which facilitate the separation of photoexcited carriers [31-33].

To visualize the separation of the carriers in the $\mathrm{M}-\mathrm{WS}_{2} / \mathrm{Te}$ device, AFM and KPFM characterizations were synchronously carried out. As depicted in Fig. 3a, the surfaces of the M-WS and Te NW are clean. Height profiles across the purple solid line and cyan solid line are shown in Fig. 3b. The purple balls indicate the smooth deformation process of $\mathrm{M}-\mathrm{WS}_{2}$, and the convex height is $253.3 \mathrm{~nm}$. The cyan balls show that the height difference between Te NW and $\mathrm{M}^{-\mathrm{WS}_{2}}$ is $75.1 \mathrm{~nm}$. This value is nearly the same as the thickness of the $\mathrm{M}-\mathrm{WS}_{2}$ (Fig. S4), confirming the seamless contact of Te NW and ${\mathrm{M}-W S_{2}}_{2}$. Fig. $3 \mathrm{c}$ displays the surface potential difference (SPD) image around the same area in Fig. 3a. Here, the SPD between the AFM tip and the contacted Te NW, convex M-WS $\left(\mathrm{Co}-\mathrm{WS}_{2}\right)$ and flat $\mathrm{M}-\mathrm{WS}_{2}(\mathrm{~F}-$ $\mathrm{WS}_{2}$ ) can be expressed as follows:

$$
\begin{aligned}
& e \mathrm{SPD}_{\mathrm{TeNW}}=\phi_{\mathrm{tip}}-\phi_{\mathrm{TeNW}}, \\
& e \mathrm{SPD}_{\mathrm{Co}-\mathrm{WS}_{2}}=\phi_{\text {tip }}-\phi_{\mathrm{Co}-\mathrm{WS}_{2}}, \\
& e \mathrm{SPD}_{\mathrm{F}-\mathrm{WS}_{2}}=\phi_{\text {tip }}-\phi_{\mathrm{F}-\mathrm{WS}_{2}},
\end{aligned}
$$

where $e$ represents the charge of an electron, $\phi_{\text {tip }}, \phi_{\mathrm{TeNW}}, \phi_{\mathrm{Co}-\mathrm{WS}_{2}}$ and $\phi_{\mathrm{F}_{-} \mathrm{WS}_{2}}$ represent the work functions of the AFM tip, Te NW,
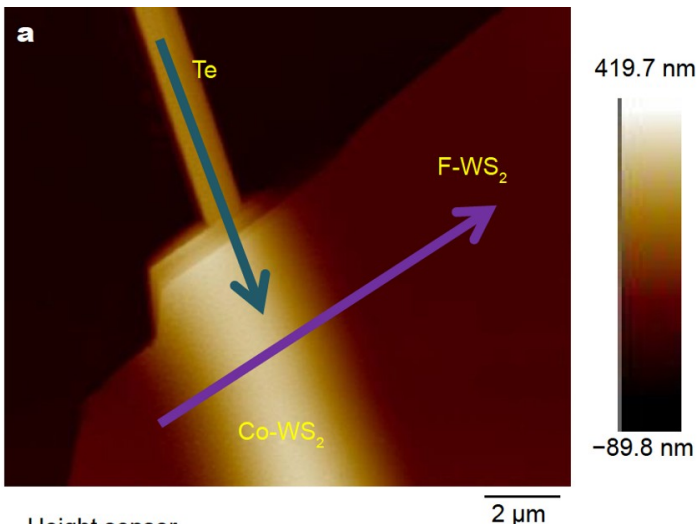

Height sensor

$2 \mu \mathrm{m}$

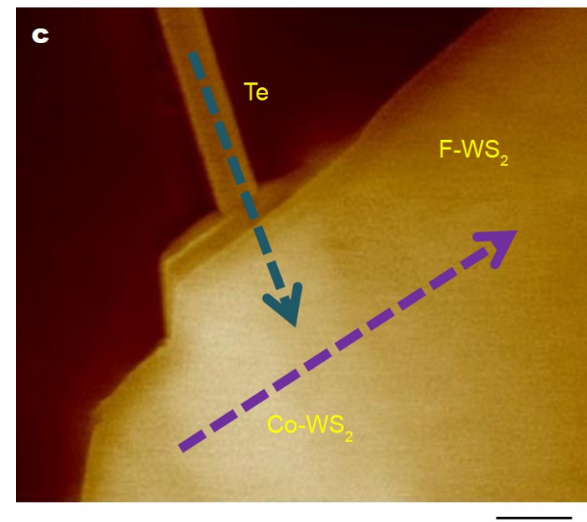

Potential

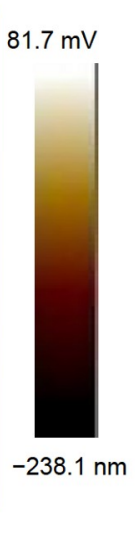

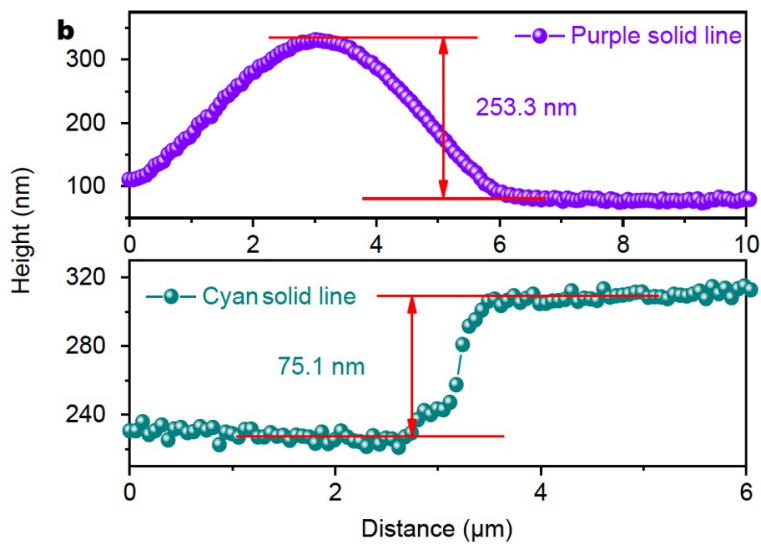

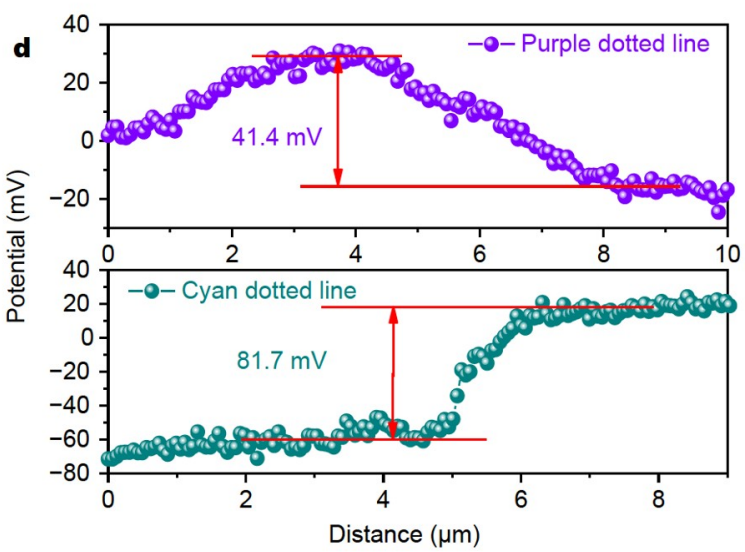

Figure 3 Electronic structure analysis of the M-WS 2 Te device. (a) AFM image around the heterojunction. (b) Plots of the thickness along the purple solid line and the cyan solid line in panel (a). (c) SPD image captured from the same area in panel (a). (d) Plots of the measured SPD along the purple dotted line and the cyan dotted line in panel (c). 
Co- $\mathrm{WS}_{2}$ and $\mathrm{F}-\mathrm{WS}_{2}$, respectively $[34,35]$. Therefore, the Fermi level difference $\left(\Delta E_{\mathrm{f}}\right)$ can be obtained according to the following formulas:

$$
\begin{aligned}
& \Delta E_{\mathrm{f} 1}=\phi_{\mathrm{F}-W S_{2}}-\phi_{\mathrm{Co}-\mathrm{WS}_{2}}=e \mathrm{SPD}_{\mathrm{Co}-\mathrm{WS}_{2}}-e \mathrm{SPD}_{\mathrm{F}_{-} \mathrm{WS}_{2}}, \\
& \Delta E_{\mathrm{f} 2}=\phi_{\mathrm{TeNW}}-\phi_{\mathrm{Co}-\mathrm{WS}_{2}}=e \mathrm{SPD}_{\mathrm{Co}-\mathrm{WS}_{2}}-e \mathrm{SPD}_{\mathrm{TeNW}} .
\end{aligned}
$$

Due to the difference in work function, different contrast areas can be clearly distinguished in Fig. 3c. As shown in Fig. 3d, the two $\Delta E_{\mathrm{f}}$ of Co-WS $\mathrm{W}_{2}$ and $\mathrm{F}-\mathrm{WS}_{2}$ (purple dotted line), Te NW and Co- $\mathrm{WS}_{2}$ (cyan dotted line) are estimated to be 41.4 and $81.7 \mathrm{meV}$, respectively. These values are higher than those of $\mathrm{WS}_{2} /$ Te device with a thin $\mathrm{WS}_{2}$ nanoflake (Fig. S5). Therefore, a thick $\mathrm{WS}_{2}$ nanoflake is chosen.

Based on the above theoretical speculation and KPFM measurement, the proposed photodetection mechanism is illustrated in Fig. 4. In the horizontal direction, as shown in Fig. 4a, Co$\mathrm{WS}_{2}$ shows a higher $E_{\mathrm{f}}$ and a smaller band gap than $\mathrm{F}-\mathrm{WS}_{2}$. Therefore, electrons will be transferred from Co-WS $\mathrm{W}_{2}$ to $\mathrm{F}-\mathrm{WS}_{2}$ until equilibrium, forming symmetrical type-II band alignments with built-in electric fields $\left(E_{1}\right.$ and $\left.E_{2}\right)$ pointing from Co-WS 2 to $\mathrm{F}_{-}-\mathrm{WS}_{2}$ (Fig. 4b). When the Co- $\mathrm{WS}_{2}$ and F-WS $\mathrm{S}_{2}$ homojunction is horizontally biased, the energy level near the drain moves downward. And the magnitude of movement is proportional to the distance from the drain. As a result, continuous type II band alignments are formed. Moreover, $E_{1}$ is forward and $E_{2}$ is reversed, forming new built-in electric fields $\left(E_{3}\right.$ and $\left.E_{4}\right)$ with the same direction. Thus, the photogenerated electron-hole pairs can be separated and transferred quickly, achieving a high photocurrent while suppressing the dark current and accelerat- ing the response speed (Fig. 4c). In the vertical direction, Co$\mathrm{WS}_{2}$ and Te NW form a heterojunction. Based on the reported results [36,37] and the KPFM analysis, Fig. $4 \mathrm{~d}$ shows the band arrangement of Co-WS $\mathrm{W}_{2}$ and Te NW. Since the difference in $E_{\mathrm{f}}$, a depletion layer with a built-in electric field $\left(E_{\mathrm{v}}\right)$ pointing from Co-WS $\mathrm{W}_{2}$ to Te NW will be formed. Driven by this $E_{\mathrm{v}}$, the photogenerated holes are trapped in the Te NW, while the photogenerated electrons are circulated in the $\mathrm{M}-\mathrm{WS}_{2}$ channel for multiple times prior to recombination, achieving a high photogain. Based on these synergetic effects, high-performance photoresponse can be expected in the $\mathrm{M}-\mathrm{WS}_{2} / \mathrm{Te}$ device.

Sequentially, the electrical and optoelectronic performance of the $\mathrm{M}-\mathrm{WS}_{2} /$ Te device was evaluated. Fig. S6 shows the transfer characteristics of individual Te NW and $\mathrm{M}-\mathrm{WS}_{2}$ devices, which verify the n-type attribute of $\mathrm{M}-\mathrm{WS}_{2}$ and the p-type attribute of Te NW. The motility of the Te NW reaches $804.9 \mathrm{~cm}^{2} \mathrm{~V}^{-1} \mathrm{~s}^{-1}$, and the current on/off ratio of $\mathrm{M}-\mathrm{WS}_{2}$ is up to $10^{5}$, further demonstrating the high quality of the samples. In the $\mathrm{M}-\mathrm{WS}_{2} / \mathrm{Te}$ device, No. 1 and No. 2 electrodes are the drain and source, respectively. Fig. 5a presents the normalized spectral photoresponse curve of the $\mathrm{M}-\mathrm{WS}_{2} / \mathrm{Te}$ device. There are two photoresponse peaks around 520 and $610 \mathrm{~nm}$, corresponding to the band structure of $\mathrm{M}-\mathrm{WS}_{2}$. Current-voltage $(I-V)$ characteristics of the $\mathrm{M}-\mathrm{WS}_{2} / \mathrm{Te}$ device under $405-\mathrm{nm}$ light with different powers are shown in Fig. 5b. Compared with that in the dark condition, the drain-source current $\left(I_{\mathrm{ds}}\right)$ increases sharply when the device is illuminated. At a bias of $1 \mathrm{~V}$, the light on/off ratio $\left(I_{\text {light }} / l_{\text {dark }}\right)$ reaches a high value of $3.4 \times 10^{4}$ (Fig. S7), exhibiting good photodetection characteristics. Fig. $5 \mathrm{c}$ summarizes the photocurrent $\left(I_{\mathrm{ph}}=I_{\text {light }}-I_{\text {dark }}\right)$ under various light intensities
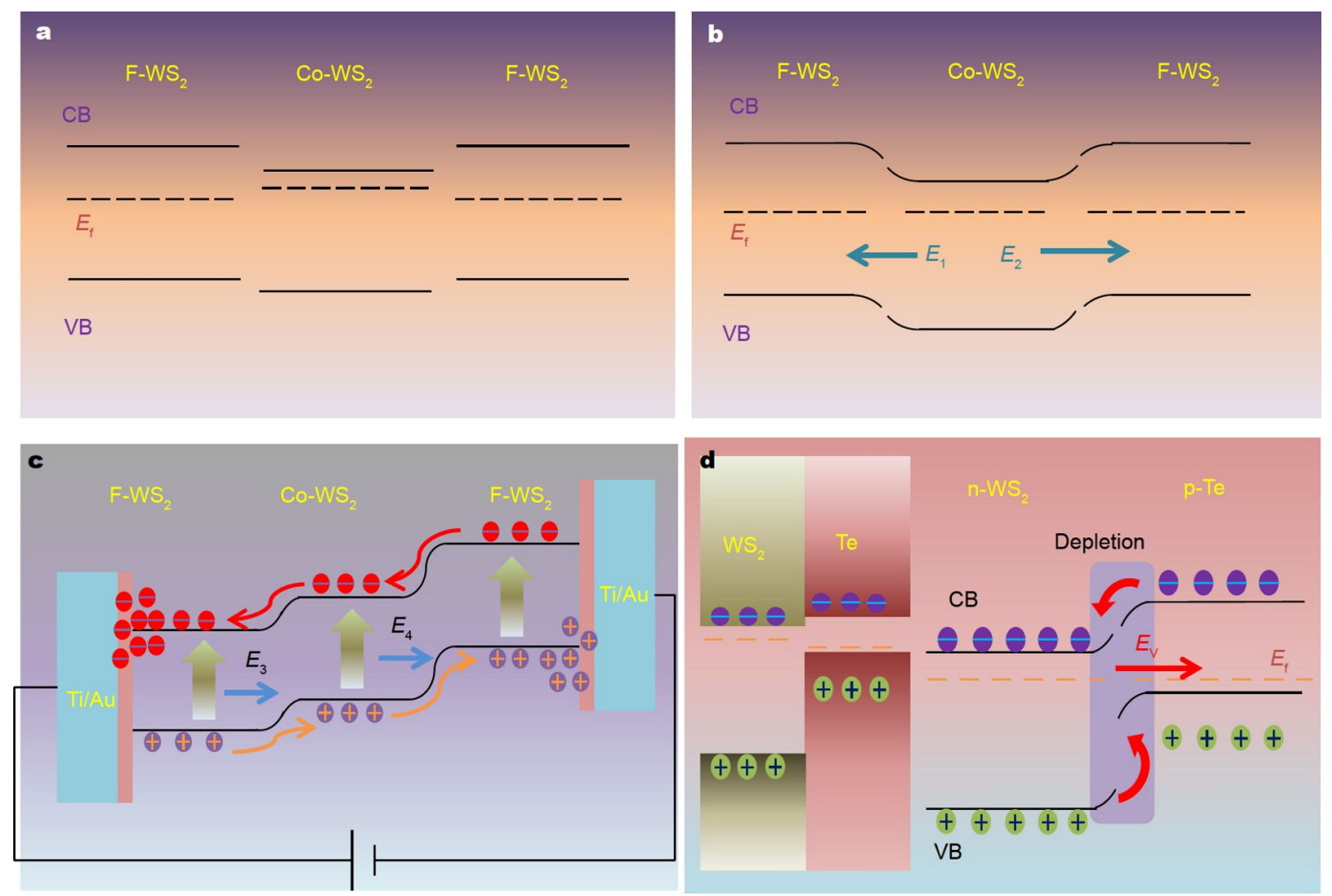

Figure 4 Photogenerated carrier transfer process in the M-WS 2 Te device. Energy band structures of F-WS $\mathrm{S}_{2}$ and Co-WS $\mathrm{S}_{2}$ (a) before and (b) after equilibrium. (c) Carrier transfer process along the horizontal direction of the F-WS $\mathrm{S}_{2}$ and Co-WS $\mathrm{S}_{2}$ homojunction after applying the bias. (d) Energy band structures of the Co- $\mathrm{WS}_{2}$ and Te heterojunction, and the corresponding carrier transfer process. 

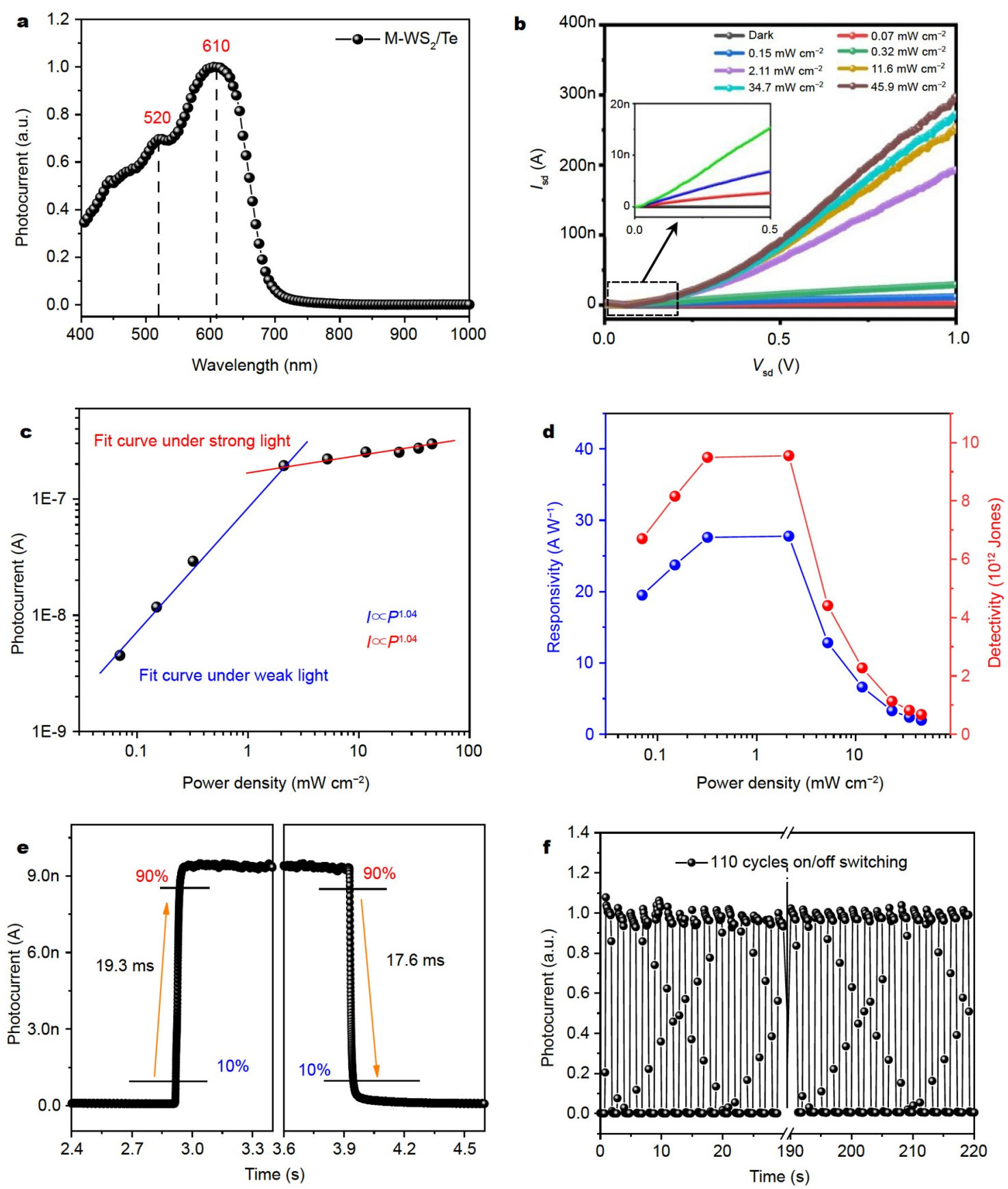

Figure 5 Optoelectronic properties of the $\mathrm{M}-\mathrm{WS}_{2} / \mathrm{Te}$ device. (a) Photoresponse spectrum of the device from 400 to $1000 \mathrm{~nm}$. (b) $I-V$ curves of the device under different light powers. Under a bias voltage of $1 \mathrm{~V}$, the incident-light-power-dependent (c) photocurrent, (d) responsivity and detectivity. (e) Temporal photoresponse under the bias of $1 \mathrm{~V}$. (f) Photoswitching characteristics of the $\mathrm{M}-\mathrm{WS}_{2} /$ Te photodetector in 110 cycles.

$(P)$. The photocurrents can be associated with a simple power law of $I_{\mathrm{ph}} \propto P^{1.04}$ for weak light (blue line), and $I_{\mathrm{ph}} \propto P^{0.14}$ for strong light (red line). Here, the difference in power exponent is related to the complex process of carriers' excitation, capture and recombination in the $\mathrm{M}-\mathrm{WS}_{2} / \mathrm{Te}$ device $[38,39]$. Compared with pure $\mathrm{M}-\mathrm{WS}_{2}$ and Te device (Figs S8-S11), the M-WS 2 /Te device exhibits suppressed dark current, and more than one order of magnitude amplified photocurrent and light on/off ratio. In addition, as presented in Fig. S12, the photocurrent of the $\mathrm{M}-\mathrm{WS}_{2} / \mathrm{Te}$ device is also much better than that of $\mathrm{M}-\mathrm{WS}_{2} / \mathrm{Te}$ vertical device (No. 1 and No. 3 electrodes as the drain and source respectively). Such enhanced photodetection perfor- mance can be interpreted by the effective separation of photogenerated electron-hole pairs under the built-in electric fields in the $\mathrm{M}-\mathrm{WS}_{2} / \mathrm{Te}$ device.

Next, to further evaluate the performance of the device, several key parameters for photodetector including photoresponsivity $\left(R_{\lambda}\right)$, detectivity $\left(D^{*}\right)$, response speed and stability were calculated. The $R_{\lambda}$ and $D^{*}$ can be extracted from the photocurrent in Fig. 5 c, using the following formulas $[40,41]$ :

$R_{\lambda}=\frac{I_{\mathrm{ph}}}{P S}$

$D^{*}=R_{\lambda} S^{1 / 2} /\left(2 e I_{\text {dark }}\right)^{1 / 2}$, 
where $S$ is the effective photosensitive area. As shown in Fig. 5d, the maximum $R_{\lambda}$ and $D^{*}$ are calculated to be $27.8 \mathrm{~A} \mathrm{~W}^{-1}$ and 9.5 $\times 10^{12}$ Jones, respectively. These values are much better than those of the ${\mathrm{M}-W S_{2}}_{2}$ device (Fig. S13). Then, photoswitching characteristics of the $\mathrm{M}-\mathrm{WS}_{2} / \mathrm{Te}$ device were investigated under various power intensities and various bias voltages. As demonstrated in Figs S14 and S15, with the increase of light intensity and the increase of bias voltage, the photocurrent exhibits an increasing tendency. This phenomenon is attributed to the fact that stronger illumination would generate more free carriers, and higher bias voltage can accelerate the separation of photogenerated carriers. In addition, as the light is turned on or off, the device quickly produces a reproducible response to the switching behavior. To evaluate the response speed of the device, transient photocurrent response was recorded using a digital oscilloscope. Fig. 5e presents a complete photoresponse cycle of the $\mathrm{M}-\mathrm{WS}_{2} / \mathrm{Te}$ device. The rise time $\left(\tau_{\text {rise }}\right)$ and decay time $\left(\tau_{\text {decay }}\right)$ are defined as the times required for the photocurrent to change from $10 \%$ to $90 \%$ and from $90 \%$ to $10 \%$, respectively $[42,43]$. Benefitting from the efficient carrier separation at the channel, fast $\tau_{\text {rise }} / \tau_{\text {decay }}$ of $19.3 / 17.6 \mathrm{~ms}$ is obtained. This response speed is also better than that of the $\mathrm{M}-\mathrm{WS}_{2}$ device (Fig. S16). Table S1 shows the comparison of important figures of merit of the relevant photodetectors. It is notable that our device stands out in terms of the overall performance, revealing the superiority of our hybrid $1 \mathrm{D} / 2 \mathrm{D}$ structure in photodetection application. Then, good stability is a prerequisite for practical application. Fig. $5 \mathrm{f}$ shows the long-term photoresponse of our device over multiple periodic light stimulations. Remarkably, even after 110 cycles of operation, the device maintains periodic photoswitching behavior, and the photocurrent fluctuation is less than $5 \%$, indicating the good stability and durability.

In addition, polarization-sensitive photodetection is one of the important functions of optoelectronic devices, and it has many applications in optical communication and remote sensing [44]. In the following step, polarized photoresponse of the $\mathrm{M}-\mathrm{WS}_{2} / \mathrm{Te}$ device was investigated. As depicted in Fig. $6 \mathrm{a}$, a polarizer and a half-wave plate are placed between the $\mathrm{M}-\mathrm{WS}_{2} / \mathrm{Te}$ device and incident light. Thus, the incident light can be converted into linearly polarized light with variable polarization direction. The orientation of Te NW and the rotation direction of linearly polarized light are labeled in Fig. 6b. Under 405-nm light illumination and $1 \mathrm{~V}$ bias, prominent anisotropic photoresponse is obtained (Fig. S17), indicating that the $\mathrm{M}-\mathrm{WS}_{2} / \mathrm{Te}$ device can recognize the differences between different polarized irradiations. To observe such photoresponse more clearly, normalized polarization angle-dependent photocurrent is extracted and plotted in a polar coordinate (Fig. 6c). These points are fitted with a sinusoidal function, which shows a two-lobed shape. The peak of photocurrent is along the $\sim 80^{\circ}$ direction, where the angle of light is polarized parallel to the Te NW. The valley of photocurrent is obtained when the light is polarized perpendicular to the Te NW. The dichroic ratio $\left(I_{\text {ph-peak }} / I_{\text {ph-valley }}\right)$ is calculated to be 2.1. This value is better than that for the thin $\mathrm{WS}_{2} /$ Te device (Fig. S18), and also better than those of many other anisotropic 2D materials like $\mathrm{GeS}_{2}$ and $\mathrm{ReS}_{2}$ [45-47]. In contrast, the $\mathrm{M}-\mathrm{WS}_{2}$ device does not show a polarization-sensitive photoresponse (Fig. S19), which is consistent with its crystal structure
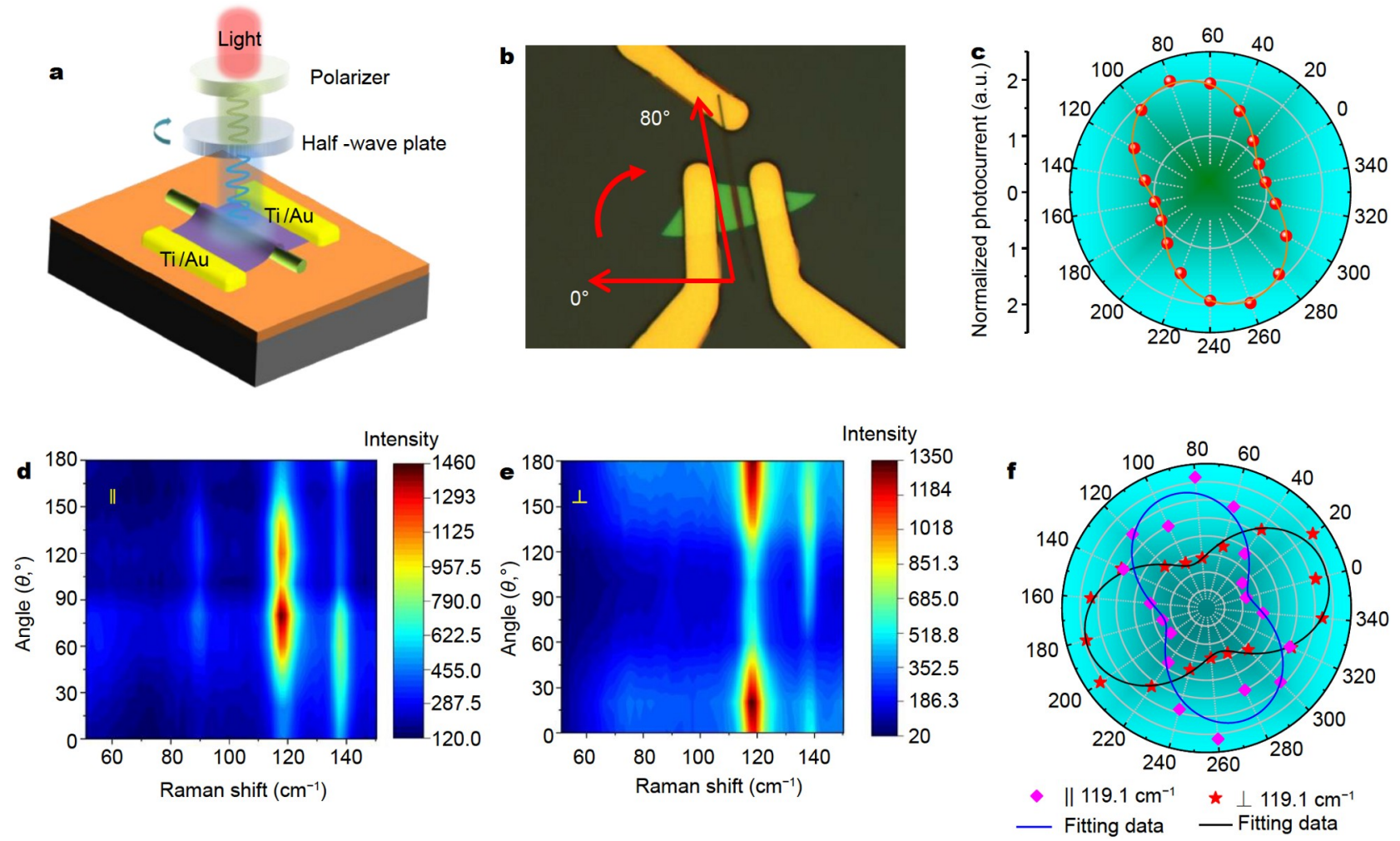

Figure 6 Polarization-sensitive detection of the $\mathrm{M}-\mathrm{Ws}_{2} / \mathrm{Te}$ device. (a) Schematic diagram of the characterization setup for the polarization-dependent measurement. (b) Optical image of the $\mathrm{M}-\mathrm{WS}_{2} / \mathrm{Te} \mathrm{NW}$ device. The orientation of the Te NW is labeled. (c) Normalized angle-resolved photocurrents in the polar coordinates, and the fitting result by a sinusoidal function. The false-color map of polarized Raman in (d) horizontal and (e) vertical configurations. (f) Polar plot of Raman intensity of $A_{1}\left(119.1 \mathrm{~cm}^{-1}\right)$. 
and geometric isotropy. Therefore, the anisotropic photodetection of the $\mathrm{M}-\mathrm{WS}_{2} / \mathrm{Te}$ may come from the Te NW. Anisotropic optical properties of the Te NW were characterized through angular-resolved polarized Raman spectroscopy. Fig. 6d, e depict the false-color map of the polarization-dependent Raman intensity under horizontal and vertical configurations, respectively. When the polarized angle is at $80^{\circ}$, the intensity of $\mathrm{A}_{1}$ peak $\left(119.1 \mathrm{~cm}^{-1}\right)$ reaches the maixmum in the horizontal configuration, while it reaches the minimum in the vertical configuration. This phenomenon corresponds to the Te NW orientation axis. Raman intensities of the $A_{1}$ peak under the two configurations are extracted and presented as a function incident angle in polar coordinates (Fig. 6f). These values can fit the sine function, and show a two-lobed shape with different polarization directions about $90^{\circ}$. Therefore, it can be inferred that the anisotropic characteristics of Te NW endow the $\mathrm{M}-\mathrm{WS}_{2} / \mathrm{Te}$ device with the ability to detect polarized light, which provides ideas for the preparation of future multifunctional optoelectronic devices.

\section{CONCLUSION}

In summary, we successfully prepare high-quality Te NWs by a PVD method, and then couple a Te NW with $\mathrm{M}-\mathrm{WS}_{2}$ to realize a hybrid $1 \mathrm{D} / 2 \mathrm{D}$ heterostructure photodetector. This structure overcomes the shortcomings of traditional 2D materials that lack effective built-in electric fields and photoconductive gain mechanism. Consequently, the constructed $\mathrm{M}-\mathrm{WS}_{2} / \mathrm{Te}$ device achieves a high photoresponsivity of $27.8 \mathrm{~A} \mathrm{~W}^{-1}$, an excellent detectivity of $9.5 \times 10^{12}$ Jones, and a short rise/decay time of 19.3/17.6 ms. These values are much better than those of an individual $\mathrm{M}-\mathrm{WS}_{2}$ device. In addition, anisotropic characteristics of Te NWs enable the polarization-sensitive photodetection of the $\mathrm{M}-\mathrm{WS}_{2} / \mathrm{Te}$ device. The dichroic ratio is up to 2.1 . This hybrid 1D/2D heterostructure inherits the characteristics of each material and expands the application range of devices, providing a promising prospect for future functional devices.

\section{Received 24 August 2021; accepted 27 October 2021;} published online 16 December 2021

1 Lopez-Sanchez O, Lembke D, Kayci M, et al. Ultrasensitive photodetectors based on monolayer $\mathrm{MoS}_{2}$. Nat Nanotech, 2013, 8: 497-501

2 Varghese A, Saha D, Thakar K, et al. Near-direct bandgap $\mathrm{WSe}_{2} / \mathrm{ReS}_{2}$ type-II pn heterojunction for enhanced ultrafast photodetection and high-performance photovoltaics. Nano Lett, 2020, 20: 1707-1717

3 Dai $\mathrm{M}$, Zheng $\mathrm{W}$, Zhang $\mathrm{X}$, et al. Enhanced piezoelectric effect derived from grain boundary in $\mathrm{MoS}_{2}$ monolayers. Nano Lett, 2020, 20: 201207

4 Cui Y, Xin R, Yu Z, et al. High-performance monolayer $\mathrm{WS}_{2}$ field-effect transistors on high- $\kappa$ dielectrics. Adv Mater, 2015, 27: 5230-5234

5 Cong C, Shang J, Wang Y, et al. Optical properties of 2D semiconductor $\mathrm{WS}_{2}$. Adv Opt Mater, 2018, 6: 1700767

6 Ye K, Liu L, Liu Y, et al. Lateral bilayer $\mathrm{MoS}_{2}-\mathrm{WS}_{2}$ heterostructure photodetectors with high responsivity and detectivity. Adv Opt Mater, 2019, 7: 1900815

7 Wang D, Zhang Z, Li B, et al. Synthesis of two-dimensional/one-dimensional heterostructures with tunable width. J Semicond, 2021, 42: 092001

8 Song Z, Wang Y, Zhu Y, et al. Targeted transfer of self-assembled CdSe nanoplatelet film onto $\mathrm{WS}_{2}$ flakes to construct hybrid heterostructures. J Semicond, 2021, 42: 082901

9 Perea-López N, Elías AL, Berkdemir A, et al. Photosensor device based on few-layered $\mathrm{WS}_{2}$ films. Adv Funct Mater, 2013, 23: 5511-5517

10 Chen Y, Gan L, Li H, et al. Achieving uniform monolayer transition metal dichalcogenides film on silicon wafer via silanization treatment:
A typical study on $\mathrm{WS}_{2}$. Adv Mater, 2017, 29: 1603550

11 Yao JD, Zheng ZQ, Shao JM, et al. Stable, highly-responsive and broadband photodetection based on large-area multilayered $\mathrm{WS}_{2}$ films grown by pulsed-laser deposition. Nanoscale, 2015, 7: 14974-14981

12 Fan Z, Chang P, Lu JG, et al. Photoluminescence and polarized photodetection of single $\mathrm{ZnO}$ nanowires. Appl Phys Lett, 2004, 85: 6128-6130

13 Fan Z, Ho JC, Jacobson ZA, et al. Large-scale, heterogeneous integration of nanowire arrays for image sensor circuitry. Proc Natl Acad Sci USA, 2008, 105: 11066-11070

14 Wang J, Gudiksen MS, Duan X, et al. Highly polarized photoluminescence and photodetection from single indium phosphide nanowires. Science, 2001, 293: 1455-1457

$15 \mathrm{Lu} \mathrm{J}$, Yao J, Yan J, et al. Strain engineering coupled with optical regulation towards a high-sensitivity $\operatorname{In}_{2} \mathrm{~S}_{3}$ photodetector. Mater Horiz, 2020, 7: 1427-1435

16 Yang S, Wang C, Sahin H, et al. Tuning the optical, magnetic, and electrical properties of $\mathrm{ReSe}_{2}$ by nanoscale strain engineering. Nano Lett, 2015, 15: 1660-1666

17 Zhu X, Lin F, Zhang Z, et al. Enhancing performance of a GaAs/ $\mathrm{AlGaAs} / \mathrm{GaAs}$ nanowire photodetector based on the two-dimensional electron-hole tube structure. Nano Lett, 2020, 20: 2654-2659

18 Ye L, Wang P, Luo W, et al. Highly polarization sensitive infrared photodetector based on black phosphorus-on-WSe $\mathrm{W}_{2}$ photogate vertical heterostructure. Nano Energy, 2017, 37: 53-60

19 Wang Q, Safdar M, Xu K, et al. Van der Waals epitaxy and photoresponse of hexagonal tellurium nanoplates on flexible mica sheets. ACS Nano, 2014, 8: 7497-7505

20 Peng H, Kioussis N, Snyder GJ. Elemental tellurium as a chiral p-type thermoelectric material. Phys Rev B, 2014, 89: 195206

21 He Z, Yang Y, Liu JW, et al. Emerging tellurium nanostructures: Controllable synthesis and their applications. Chem Soc Rev, 2017, 46: 2732-2753

22 Nayak PK, Yeh CH, Chen YC, et al. Layer-dependent optical conductivity in atomic thin $\mathrm{WS}_{2}$ by reflection contrast spectroscopy. ACS Appl Mater Interfaces, 2014, 6: 16020-16026

23 Reed EJ. Two-dimensional tellurium. Nature, 2017, 552: 40-41

24 Li H, Contryman AW, Qian X, et al. Optoelectronic crystal of artificial atoms in strain-textured molybdenum disulphide. Nat Commun, 2015, 6: 7381

25 Lloyd D, Liu X, Christopher JW, et al. Band gap engineering with ultralarge biaxial strains in suspended monolayer $\mathrm{MoS}_{2}$. Nano Lett, 2016, 16: 5836-5841

26 Duan X, Wang C, Shaw JC, et al. Lateral epitaxial growth of twodimensional layered semiconductor heterojunctions. Nat Nanotech, 2014, 9: 1024-1030

27 Ma C, Shi $\mathrm{Y}, \mathrm{Hu} \mathrm{W}$, et al. Heterostructured $\mathrm{WS}_{2} / \mathrm{CH}_{3} \mathrm{NH}_{3} \mathrm{PbI}_{3}$ photoconductors with suppressed dark current and enhanced photodetectivity. Adv Mater, 2016, 28: 3683-3689

28 Xue Y, Zhang Y, Liu Y, et al. Scalable production of a few-layer $\mathrm{MoS}_{2} /$ $\mathrm{WS}_{2}$ vertical heterojunction array and its application for photodetectors. ACS Nano, 2016, 10: 573-580

$29 \mathrm{Lu}$ J, Zheng Z, Yao J, et al. $2 \mathrm{D} \mathrm{In}_{2} \mathrm{~S}_{3}$ nanoflake coupled with graphene toward high-sensitivity and fast-response bulk-silicon schottky photodetector. Small, 2019, 15: 1904912

30 Conley HJ, Wang B, Ziegler JI, et al. Bandgap engineering of strained monolayer and bilayer $\mathrm{MoS}_{2}$. Nano Lett, 2013, 13: 3626-3630

31 Gao W, Zheng Z, Huang L, et al. Self-powered $\mathrm{SnS}_{1-x} \mathrm{Se}_{x}$ alloy/silicon heterojunction photodetectors with high sensitivity in a wide spectral range. ACS Appl Mater Interfaces, 2019, 11: 40222-40231

32 Zhou $\mathrm{Y}$, Zhang L, Gao W, et al. A reasonably designed 2D $\mathrm{WS}_{2}$ and CdS microwire heterojunction for high performance photoresponse. Nanoscale, 2021, 13: 5660-5669

33 Wang X, Pan L, Yang J, et al. Direct synthesis and enhanced rectification of alloy-to-alloy 2D type-II $\mathrm{MoS}_{2(1-x)} \mathrm{Se}_{2 x} / \mathrm{SnS}_{2(1-y)} \mathrm{Se}_{2 y}$ heterostructures. Adv Mater, 2021, 33: 2006908

34 Zheng Z, Yao J, Xiao J, et al. Synergistic effect of hybrid multilayer $\mathrm{In}_{2} \mathrm{Se}_{3}$ and nanodiamonds for highly sensitive photodetectors. ACS Appl Mater Interfaces, 2016, 8: 20200-20211 
35 Zheng Z, Yao J, Yang G. Self-assembly of the lateral $\mathrm{In}_{2} \mathrm{Se}_{3} / \mathrm{CuInSe}_{2}$ heterojunction for enhanced photodetection. ACS Appl Mater Interfaces, 2017, 9: 7288-7296

36 Tao JJ, Jiang J, Zhao SN, et al. Fabrication of 1D Te/2D ReS ${ }_{2}$ mixeddimensional van der Waals p-n heterojunction for high-performance phototransistor. ACS Nano, 2021, 15: 3241-3250

37 Huo N, Kang J, Wei Z, et al. Novel and enhanced optoelectronic performances of multilayer $\mathrm{MoS}_{2}-\mathrm{WS}_{2}$ heterostructure transistors. Adv Funct Mater, 2014, 24: 7025-7031

38 Yim C, Lee K, McEvoy N, et al. High-performance hybrid electronic devices from layered $\mathrm{PtSe}_{2}$ films grown at low temperature. ACS Nano, 2016, 10: 9550-9558

39 Gong M, Liu Q, Cook B, et al. All-printable $\mathrm{ZnO}$ quantum dots/graphene van der Waals heterostructures for ultrasensitive detection of ultraviolet light. ACS Nano, 2017, 11: 4114-4123

40 Gao L, Zeng K, Guo J, et al. Passivated single-crystalline $\mathrm{CH}_{3} \mathrm{NH}_{3} \mathrm{PbI}_{3}$ nanowire photodetector with high detectivity and polarization sensitivity. Nano Lett, 2016, 16: 7446-7454

$41 \mathrm{Lu}$ J, Yan J, Yao J, et al. All-dielectric nanostructure Fabry-Pérot-enhanced Mie resonances coupled with photogain modulation toward ultrasensitive $\operatorname{In}_{2} \mathrm{~S}_{3}$ photodetector. Adv Funct Mater, 2021, 31: 2007987

42 Yang $\mathrm{M}, \mathrm{Gao} \mathrm{W}$, Song $\mathrm{Q}$, et al. Universal strategy integrating strain and interface engineering to drive high-performance $2 \mathrm{D}$ material photodetectors. Adv Opt Mater, 2021, 9: 2100450

43 Gong C, Chu J, Yin C, et al. Self-confined growth of ultrathin 2D nonlayered wide-bandgap semiconductor $\mathrm{CuBr}$ flakes. Adv Mater, 2019, 31: 1903580

44 Tyo JS, Goldstein DL, Chenault DB, et al. Review of passive imaging polarimetry for remote sensing applications. Appl Opt, 2006, 45: 54535469

45 Yang Y, Liu SC, Wang X, et al. Polarization-sensitive ultraviolet photodetection of anisotropic 2D GeS 2 . Adv Funct Mater, 2019, 29: 1900411

46 Liu F, Zheng S, He X, et al. Highly sensitive detection of polarized light using anisotropic 2D ReS 2 . Adv Funct Mater, 2016, 26: 1169-1177

47 Pi L, Hu C, Shen W, et al. Highly in-plane anisotropic $2 \mathrm{D} \mathrm{PdSe}_{2}$ for polarized photodetection with orientation selectivity. Adv Funct Mater, 2020, 31: 2006774

Acknowledgements This work was supported by the National Natural Science Foundation of China $(61805044,62004071$ and 11674310), the Key Platforms and Research Projects of Department of Education of Guangdong Province (2018KTSCX050), Guangdong Provincial Key Laboratory of Information Photonics Technology (2020B121201011), and "The Pearl River Talent Recruitment Program" (2019ZT08X639). The authors thank Lin Liu from the State Key Laboratory of Optoelectronic Materials and Technologies, Sun Yat-sen University for the device constructing.

Author contributions Zheng $\mathrm{Z}$ and $\mathrm{Li} \mathrm{J}$ designed the project and the experiment. Zhou $\mathrm{Y}$ performed the main experiments and wrote this manuscript. Han L, Song Q and Gao W completed the rest of the experiment and helped write this manuscript. The other authors helped to analyze the data, discussed the results and contributed to the theoretical analysis. All authors contributed to the general discussion.

Conflict of interest The authors declare that they have no conflict of interest.

Supplementary information Experimental details and supporting data are available in the online version of the paper.

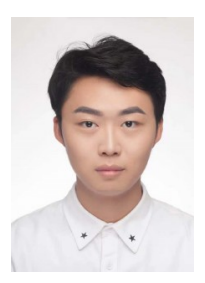

Yuchen Zhou is a master degree candidate at the school of Materials and Energy, Guangdong University of Technology. He received his BSc degree from Guilin University of Electronic Technology in 2019. His current research focuses on designing intelligent $2 \mathrm{D}$ material devices for photodetection applications.

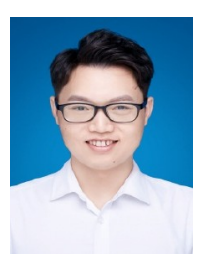

Zhaoqiang Zheng received his BSc degree from Hunan University (2011) and $\mathrm{PhD}$ degree from Sun Yat-sen University (2017). Then, he joined the School of Materials and Energy, Guangdong University of Technology and currently is an associate professor. His research interests are the design, synthesis and photodetection applications of novel $2 \mathrm{D}$ materials and their heterostructures.

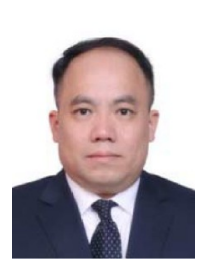

Jingbo $\mathrm{Li}$ received his $\mathrm{PhD}$ degree from the Institute of Semiconductors, Chinese Academy of Sciences (2001) Then, he spent six years at Lawrence Berkeley National Laboratory. From 2007 to 2019, he worked as a professor at the Institute of Semiconductors, Chinese Academy of Sciences. Since 2019, he has been a professor and the dean of Institute of Semiconductors, South China Normal University. His research interests include the design, fabrication, and application of novel nanostructured semiconductors.

\section{混合1D/2D异质结构耦合电子结构工程用于高灵敏 度和偏振依赖的光电探测器}

周瑜琛 ${ }^{1}$, 韩理想 ${ }^{1}$, 宋琦琦 ${ }^{1}$, 高伟 ${ }^{2}$, 杨孟孟 ${ }^{1}$, 郑照强 ${ }^{1^{*}}$, 黄乐 ${ }^{1}$, 姚健东 ${ }^{3}$, 李京波 $2,4,5^{*}$

摘要 随着光电探测器的广泛应用, 人们迫切需要高灵敏度、偏振依 赖的光电探测技术. 在这一领域, 二维二硫化铇 $(2 \mathrm{D} \mathrm{WS}$ ) 表现出出色的 光学和电子特性, 使其成为一种在光电应用领域有吸引力的光敏材料. 但是, $2 \mathrm{D} \mathrm{WS}_{2}$ 缺乏有效的内建电场和光电导增益机制, 阻碍了其在高 性能光电探测器中的应用. 在此, 我们提出了一种包含 $1 \mathrm{D}$ Te和 $2 \mathrm{D} \mathrm{WS}_{2}$ 的混合异质结构光电探测器. 在该器件中, 二维 $\mathrm{WS}_{2}$ 在 $1 \mathrm{D} \mathrm{Te}$ 上产生平 面内形态应变, 该应变能调节 $\mathrm{WS}_{2}$ 的局部电子结构, 并在水平方向上形 成II型能带排列. 此外, $2 \mathrm{D} \mathrm{WS}$ 和 $1 \mathrm{D} \mathrm{Te}$ 的垂直异质结引入了光电导增 益. 这两种效应优化了光致载流子的转移, 使得光电探测器具有较高的 灵敏度 (光响应度为 $27.7 \mathrm{~A} \mathrm{~W}^{-1}$, 探测度为 $9.5 \times 10^{12}$ Jones, 上升/衰减 时间为 $19.3 / 17.6 \mathrm{~ms})$. 此外, 该器件还获得了各向异性的光电探测特性, 其二向色比可达 2.1 . 这种混合的 $1 \mathrm{D} / 2 \mathrm{D}$ 异质结构克服了单种材料固有 的局限性, 实现了新的性能, 为构建多功能光电器件开辟了新的途径. 